\title{
NONLINEAR EVOLUTION EQUATIONS ON BANACH SPACE1
}

\author{
N.U. AHMED \\ University of Ottawa \\ Department of Mathematics and Department of Electrical Engineering \\ Ottawa, CANADA
}

\begin{abstract}
In this paper we consider the questions of existence and uniqueness of solutions of certain semilinear and quasilinear evolution equations on Banach space. We consider both deterministic and stochastic systems. The approach is based on semigroup theory and fixed point theorems. Our results allow the nonlinear perturbations in all the semilinear problems to be bounded or unbounded with reference to the base space, thereby increasing the scope for applications to partial differential equations. Further, quasilinear stochastic evolution equations seemingly have never been considered in the literature.
\end{abstract}

Key words: Evolution equations, deterministic, stochastic, semilinear, quasilinear, integral equations, semigroup theory, Wiener process.

AMS (MOS) subject classification: 34G05, 35A05, 93C25, $93 \mathrm{E} 03$.

\section{SEMILINEAR EVOLUTION EQUATIONS (DETERMINISTIC).}

In this section we consider the questions of existence of solutions of certain semilinear and quaslinear evolution equations on Banach space. First we wish to consider the semilinear evolution equation,

$$
\begin{gathered}
\left(\frac{d}{d t}\right) y+A(t) y=f(t, y), t \in(0, a] \\
y(0)=y_{0} .
\end{gathered}
$$

This can be written as a nonlinear Volterra integral equation,

$$
y(t)=v(t)+\int_{0}^{t} U(t, \tau) f(\tau, y(\tau)) d \tau, t \in I
$$

where $U$ is the evolution operator corresponding to $A$.

\footnotetext{
${ }^{1}$ Received: February, 1991. Revised: May, 1991.
} 
In fact a solution of the integral equation (1.2) is a mild solution of the Cauchy problem (1.1).

Let $H$ be a Hilbert space and $V$ a reflexive Banach space with the embedding $V \hookrightarrow H$ being continuous and dense. Identifying $H$ with its dual, we have $V \hookrightarrow H \hookrightarrow V^{*}$ where $V^{*}$ is the dual of $V$. For $1<p, q<\infty$, satisfying $(1 / p)+(1 / q)=1$, and $I$ a finite interval $[0, a]$, let $Z \equiv L_{p}(I, V)$ with dual given by $Z^{*} \equiv L_{q}\left(I, V^{*}\right)$. Since $V$ is reflexive, these spaces are also reflexive Banach spaces. We shall need the following well-known result (see Tanabe [11]).

Lemma 1: Suppose the operator valued function $A \equiv\{A(t), t \in I\}$ satisfies the following conditions:

$\left(A_{1}\right) \quad A \in L_{\infty}\left(I, \mathcal{L}\left(V, V^{*}\right)\right) \cap C^{\nu}\left(I, \mathcal{L}\left(V, V^{*}\right)\right)$ for some $\nu \in(0,1)$.

$\left(A_{2}\right)$ : There exist $\lambda \geq 0, \beta>0$ such that

$$
(A(t) \xi, \xi)_{V^{*}, V}+\lambda\|\xi\|_{H}^{2} \geq \beta\|\xi\|_{V}^{2} \text { for all } \xi \in V .
$$

Then - A generates an evolution operator $U(t, \tau), 0 \leq \tau \leq t \leq a$ which is strongly continuous on $\Delta \equiv\{(t, \tau): 0 \leq \tau \leq t \leq a\}$ both in $H$ and $V^{*}$ and there exists a constant $c>0$ such that

$$
\begin{gathered}
\|U(t, \tau)\|_{\mathcal{L}(H)} \leq c,\|U(t, \tau)\|_{\mathcal{L}\left(V^{*}\right)} \leq c, \\
\|U(t, \tau)\|_{\mathcal{L}(H, V)} \leq c / \sqrt{(t-\tau)}, \quad\|U(t, \tau)\|_{\mathcal{L}\left(V^{*}, H\right)} \leq c / \sqrt{(t-\tau)}
\end{gathered}
$$

and hence

$$
\|U(t, \tau)\|_{\mathcal{L}\left(V^{*}, V\right)} \leq c /(t-\tau)
$$

According to this lemma, it follows from the variation of constants formula that the evolution equation (1.1) can be written as the integral equation (1.2) with $v(t) \equiv U(t, 0) y_{0}$. Hence a solution of the integral equation is a mild solution of the differential equation (1.1).

Note that there are other types of conditions for existence of the evolution operator $U$ (see [2], [9], [10], [11]).

We prove the following result without imposing the standard Lipschitz and linear growth assumptions on $f$. However, we assume that $f$ satisfies the Caratheödory property in the sense of $\left(a_{2}\right)$ given below.

Theorem 2: $\quad$ Suppose the operators $A$ and $f$ satisfy the following assumptions:

$\left(a_{1}\right)$ : The operator $A$ satisfies the assumptions $\left(A_{1}\right)$ and $\left(A_{2}\right)$ of Lemma 1 and there exists a family of reflexive Banach spaces $V^{\alpha}, 0 \leq \alpha \leq 1$, with $V^{0} \equiv H, V^{1} \equiv V$ and duals 
$\left(V^{\alpha}\right)^{*}=V^{-\alpha}$ so that for $0<\alpha<1$,

$$
V\left\llcornerV ^ { \alpha } \left\llcornerH \left\llcornerV ^ { - \alpha } \left\llcorner V^{*}\right.\right.\right.\right.
$$

with the embeddings being continuous, dense and compact.

$\left(a_{2}\right): \xi \rightarrow f(t, \xi)$ is continuous from $V^{\alpha}$ to $V^{-\alpha}$ and $t \rightarrow f(t, \xi)$ is strongly measurable from $I$ to $V^{-\alpha}$.

$\left(a_{3}\right):$ There exist $h \in L_{q}\left(I, R^{+}\right), 0 \leq \gamma<\infty$ and $1<p<(1 / \alpha)$ satisfying $(1 / p)+(1 / q)=1$, such that

$$
\|f(t, \xi)\|_{V^{-\alpha}} \leq h(t)+\eta\left(\|\xi\|_{V^{\alpha}}\right)^{p / q} \text { for all } \xi \in V^{\alpha} \text {. }
$$

Then for every $y_{0} \in V^{-\alpha}$ the Cauchy problem (1.2) has a solution $y \in L_{p}\left(I, V^{\alpha}\right) \cap C\left((0, a), V^{\alpha}\right)$ for sufficiently small a. Further, for $y_{0} \in V^{\alpha}$, the solution $y \in C\left(I, V^{\alpha}\right)$.

Proof: $\quad$ For the fixed $y_{0}$ define the operator $G$ by

$$
(G \xi)(t) \equiv U(t, 0) y_{0}+\int_{0}^{t} U(t, s) f(s, \xi(s)) d s, t \in I .
$$

Let $Z$ denote $L_{p}\left(I, V^{\alpha}\right)$ and $Z^{*}$ its dual $L_{q}\left(I, V^{-\alpha}\right)$ and $F$ the nonlinear operator $(F \xi)(t) \equiv f(t, \xi(t))$. Under the assumptions $\left(a_{2}\right)$ and $\left(a_{3}\right)$, the operator $F$ is continuous and maps bounded sets of $Z$ into bounded sets of $Z^{*}$ (see [1], Lemma 1, p. 4). By virtue of assumption $\left(a_{1}\right)$, it follows from Lemma 1 that, for $-1 \leq \beta<\alpha \leq 1$, $\|U(t, \tau)\|_{\mathcal{L}\left(V^{\beta}, V^{\alpha}\right)} \leq c /(t-\tau)^{(\alpha-\beta) / 2}$ for some constant $c=c(\alpha, \beta)>0$. Thus the linear operator $U$, given by $(U \varphi)(t) \equiv \int_{0}^{t} U(t, s) \varphi(s) d s$, maps $Z^{*}$ into $Z$ and hence $G$ maps $Z$ into itself and one can verify that, for $\xi \in B_{r}$, a ball of radius $r$ in $L_{p}\left(I, V^{\alpha}\right)$,

$$
\|G \xi\|_{Z} \leq(a)^{((1-\alpha p) / p)}\left\{k_{1}+k_{2}(a)^{(\alpha+(1-\alpha p))}\right\},
$$

where $k_{1}$ is a constant depending on $c, \alpha, p$ and $\left\|y_{0}\right\|_{V-\alpha}$; and $k_{2}$ is another constant dependent on $c, \alpha, p$ and $r$. Hence, for every $r>0$, there exists a constant $a_{r}$ such that for $a \equiv a_{r}, G B_{r} \subseteq B_{r}$. We show that $G B_{r}$ is conditionally compact subset of $B_{r}$. Indeed, for $g \in F B_{r}$ and $\epsilon>0$ satisfying $0<\alpha+\epsilon<(1 / p)$,

$$
\begin{gathered}
\|W(t, g)\|_{V^{\alpha+2 \epsilon}}=\left\|\int_{0}^{t} U(t, s) g(s) d s\right\|_{V^{\alpha+2 \epsilon}} \\
\leq(c \bar{r})\left(t^{1-(\alpha+\epsilon) p} /(1-(\alpha+\epsilon) p)\right)^{(1 / p)}<\infty
\end{gathered}
$$




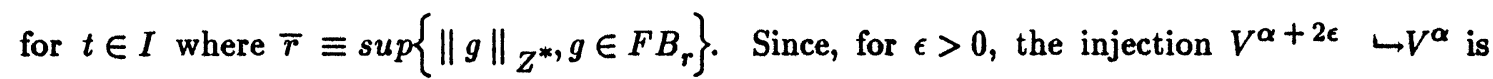
compact, it follows from this that $W\left(t, F B_{r}\right)$ is a compact subset of $V^{\alpha}$. Hence, by virtue of strong continuity of the evolution operator $U$ and the Lebesgue dominated convergence theorem, we have

$$
\lim _{h \backslash 0} \int_{I \cap(I-h)}\left(\|(U(t+h, t)-I) W(t, g)\|_{V^{\alpha}}\right)^{p} d t=0
$$

uniformly in $g \in F B_{r}$. Further, it follows from Hölder's inequality that, for $g \in F B_{r}$,

$$
\left\|\int_{t}^{t+h} U(t+h, s) g(s) d s\right\|_{V^{\alpha}} \leq \tilde{C}(h)^{((1 / p)-\alpha)}
$$

and hence

$$
\lim _{h \downarrow 0} \int_{I \cap(I-h)}\left(\left\|\int_{t}^{t+h} U(t+h, s) g(s) d s\right\|_{V^{\alpha}}\right)^{p} d t=0
$$

uniformly in $g \in F B_{r}$. Similarly, for $y_{0} \in V^{-\alpha}$, we have

$$
\lim _{h \backslash 0} \int_{I \cap(I-h)}\left(\left\|(U(t+h, 0)-U(t, 0)) y_{0}\right\|_{V^{\alpha}}\right)^{p} d t=0
$$

Thus it follows from $(1.8)-(1.11)$ that

$$
\lim _{h \downarrow 0} \int_{I \cap(I-h)}\left(\|y(t+h)-y(t)\|_{V^{\alpha}}\right)^{p} d t=0
$$

uniformly with respect to $y \in G B_{r} \subseteq B_{r} \subset L_{p}\left(I, V^{\alpha}\right)$. One can also verify relations similar to (1.8) $-(1.11)$ for $t-h \geq 0$, and hence we have

$$
\lim _{h \downarrow 0} \int_{I \cap(I+h)}\left(\|y(t)-y(t-h)\|_{V^{\alpha}}\right)^{p} d t=0
$$

uniformly with respect to $y \in G B_{r}$. Thus $G B_{r}$ is a conditionally compact subset of $B_{r}$ and hence, by Schauder's fixed point theorem, $G$ has a fixed point in $B_{r}$. The last part of the conclusion follows from strong continuity of $U(t, \tau)$.

Remark 3: $\quad$ According to our assumptions $\left(a_{2}\right)$ and $\left(a_{3}\right), f$ represents a nonlinear differential operator admitting polynomial growth. To admit stronger nonlinearities, one needs Orliczs-Sobolev spaces. 
Next we present a result involving local Lipschitz property.

\section{Theorem 4: $\quad$ Consider the evolution equation (1.2) and suppose}

$\left(a_{1}\right)$ : A satisfies the assumptions of Lemma 1 .

$\left(a_{2}\right): f: I \times H \rightarrow V^{*}$, and, for each $r>0$, and $y_{0} \in H$, there exists a constant $K_{r}$ such that

$$
\|f(t, \xi)-f(t, \eta)\|_{V^{*}} \leq K_{r}\|\xi-\eta\|_{H}
$$

and

$$
\|f(t, \xi)\|_{V^{*}} \leq K_{r}\left(1+\|\xi\|_{H}\right),
$$

for all $t \in I \equiv[0, a]$, and $\xi, \eta \in B_{r}\left(y_{0}\right) \equiv\left\{\zeta \in H:\left\|\zeta-y_{0}\right\|_{H} \leq r\right\}$. Then there exists $a_{r} \in(0, a]$ so that the problem (1.2) has a unique mild solution $y \in C\left(I_{r}, H\right)$ where $I_{r} \equiv\left[0, a_{r}\right]$.

Proof: Let $\xi \in C(I, H)$ satisfying $\xi(0)=y_{0}$ and $\xi(t) \in B_{r}\left(y_{0}\right)$ for all $t \in I$. Define the operator $G$ by

$$
(G \xi)(t) \equiv U(t, 0) y_{0}+\int_{0}^{t} U(t, s) f(s, \xi(s)) d s, \text { for } t \in I .
$$

Since $U(t, s)$ is strongly continuous on $\Delta \equiv\{0 \leq s \leq t \leq a\}$ in $\mathcal{L}(H)$, there exists a $\tau \in I$ such that $\left\|U(t, 0) y_{0}-y_{0}\right\|_{H} \leq(r / 2)$ for $0 \leq t \leq \tau$. Further, it follows from assumption $\left(a_{2}\right)$ and the estimate (1.3) that

$$
\begin{gathered}
\left\|\int_{0}^{t} U(t, s) f(s, \xi(s)) d s\right\|_{H} \leq 2 c K_{r}\left(1+\sup _{0 \leq s \leq t}\|\xi(s)\|_{H}\right) t^{(1 / 2)} \\
\leq 2 c K_{r}\left(1+r+\left\|y_{0}\right\|_{H}\right) t^{(1 / 2)}
\end{gathered}
$$

Hence there exists $\sigma \in I$ such that

$$
\left\|\int_{0}^{t} U(t, s) f(s, \xi(s)) d s\right\|_{H} \leq(r / 2) \text { for } 0 \leq t \leq \sigma .
$$

Thus, for $a_{r} \equiv \min \{r, \sigma\}$, and for $t \in I_{r} \equiv\left[0, a_{r}\right]$, we have $\left\|(G \xi)(t)-y_{0}\right\|_{H} \leq r$. Further, $t \rightarrow(G \xi)(t)$ is continuous $H$-valued function on $I$. Defining

$$
X_{r} \equiv\left\{x \in C\left(I_{r}, H\right): x(0)=y_{0} \text { and } x(t) \in B_{r}\left(y_{0}\right) \text { for } t \in I_{r}\right\}
$$

we have $G: X_{r} \rightarrow X_{r}$, and, for $\xi, \zeta \in X_{r}$, it follows from Lemma 1 that

$$
\|(G \xi)(t)-(G \zeta)(t)\|_{H} \leq c K_{r} \int_{0}^{t}(1 / \sqrt{(t-s)})\|\xi(s)-\zeta(s)\|_{H} d s .
$$


Iterating this $n$ times, for $n$ sufficiently large, one can verify that the $n$-fold composition $G^{n}$ is a contraction in $X_{r}$. Since $X_{r}$ is a closed subset of $C\left(I_{r}, H\right)$ and $G^{n}$ is a contraction in $X_{r}$, it follows from Banach fixed point theorem that $G^{n}$, and hence, $G$ has a unique fixed point in $X_{r}$. This proves that the Cauchy problem (1.2) has a unique (local) mild solution.

Next, we consider a system governed by an integro-differential equation of the form,

$$
\begin{gathered}
(d / d t) x=-A x(t)+f(x(t))+\int_{-a}^{t} h(t-s) g(x(s)) d s, t \in[0, b] \\
x(t)=\varphi(t), t \in[-a, 0], 0<a, b<\infty
\end{gathered}
$$

in a Banach space $X$ where $-A$ is merely the infinitesimal generator of a semigroup in $X$.

Theorem 5: $\quad$ Suppose the following conditions hold:

$\left(a_{1}\right): \quad-A$ is the infinitesimal generator of an analytic semigroup $T(t), t \geq 0$, in $X$.

$\left(a_{2}\right): \quad 0 \leq \alpha<1 ; X_{\alpha} \equiv\left[D\left(A^{\alpha}\right)\right]$ is the Banach space with respect to the graph topology induced by the graph norm given by $\|\zeta\|_{\alpha} \equiv\left\|A^{\alpha} \zeta\right\|+\|\zeta\|$ for $\zeta \in D\left(A^{\alpha}\right)$.

$\left(a_{3}\right)$ : The functions $f$ and $g$ map $X_{\alpha}$ to $X$ and there exists a constant $C>0$ such that for $q \equiv f, g$,

$$
\begin{gathered}
\|q(\xi)-q(\zeta)\|_{X} \leq C\|\xi-\zeta\|_{\alpha} \text { and } \\
\|q(\zeta)\|_{X} \leq C\left(1+\|\zeta\|_{\alpha}\right) \text { for all } \xi, \zeta \in X_{\alpha} .
\end{gathered}
$$

$\left(a_{4}\right): \quad h \in L_{1}([0, a+b], R)$.

Then, for every $\varphi \in C\left([-a, 0], X_{\alpha}\right)$, the evolution equation (1.15) has a unique mild solution $x \in C\left([-a, b], X_{\alpha}\right)$.

Proof: Define the operator $G$ on $C\left([-a, b], X_{\alpha}\right)$ by

$$
\begin{gathered}
(G x)(t) \equiv T(t) \varphi(0)+\int_{0}^{t} T(t-s) f(x(s)) d s \\
+\int_{0}^{t} T(t-\tau)\left(\int_{-a}^{\tau} h(\tau-s) g(x(s)) d s\right) d \tau
\end{gathered}
$$

where $T(t), t \geq 0$, is the semigroup corresponding to the generator $-A$. Using the assumptions and the facts that $\varphi \in C\left([-a, 0], X_{\alpha}\right)$ and that, for analytic semigroups, there exists a constant $C_{\alpha}$ such that $\left\|A^{\alpha} T(t)\right\|_{\mathcal{L}(X)} \leq\left(C_{\alpha} / t^{\alpha}\right)$ for $t>0$, one can verify that $G$ maps $C\left([-a, b], X_{\alpha}\right)$ to itself. Then, for any pair $x, y \in C\left([-a, b], X_{\alpha}\right)$ satisfying $x(s)=y(s)=\rho(s)$ for $s \in[-a, 0]$, define 


$$
\begin{aligned}
\rho_{t}(x, y) & \equiv \sup \left\{\left\|A^{\alpha} x(s)-A^{\alpha} y(s)\right\|_{X}, 0 \leq s \leq t\right\} \\
& \equiv \sup \left\{\|x(s)-y(s)\|_{\alpha}, 0 \leq s \leq t\right\} .
\end{aligned}
$$

After some computations, involving (1.16) and the given assumptions, one arrives at the following inequality,

$$
\rho_{t}(G x, G y) \leq L \int_{0}^{t}\left(1 /(t-s)^{\alpha}\right) \rho_{s}(x, y) d s, t \in[0, b],
$$

where $L \equiv C C_{\alpha}(1+\bar{h})$, and $\bar{h} \equiv \int_{0}^{a+b}|h(t)| d t$.

By repeated substitution of (1.17) into itself, after $n$ steps we obtain

$$
\rho_{b}\left(G^{n} x, G^{n} y\right) \leq L_{n} \rho_{b}(x, y),
$$

where $L_{n}$ is a constant depending only on $L, \alpha$, and $b$. For $n$ sufficiently large, $0 \leq L_{n}<1$; and hence $G^{n}$ is a contraction in $C\left([0, b], X_{\alpha}\right)$. Thus $G^{n}$, and hence $G$, has a unique fixed point in $C\left([-a, b], X_{\alpha}\right)$.

It is clear from the proof that this result also holds for operator valued functions $h \in L_{1}([0, a+b], \mathcal{L}(X))$. For linear evolution equations, DaPrato admits even more general operator valued functions $h$ (see [6]).

\section{SEMILINEAR AND QUASILINEAR EVOLUTION EQUATIONS (STOCHASTIC).}

Consider the stochastic evolution equation

$$
\begin{gathered}
d x+A x d t=f(x) d t+\sigma(x) d W, t \in I \equiv[0, a] \\
x(0)=x_{0} .
\end{gathered}
$$

Let $X$ be a Hilbert space and $F$ another Hilbert space which we assume to be separable. Let $\left(\Omega, \Im, \Im_{t}, t \geq 0, P\right)$ be a complete probability space furnished with a complete family of right continuous increasing $\sigma$-algebras $\left\{\Im_{t}, t \geq 0\right\}$ satisfying $\Im_{t} \subset \Im$ for $t \geq 0$. The process $\{W(t), t \geq 0\}$ is an $F$-valued $\Im_{t}$-adapted Brownian motion with $P\{W(0)=0\}=1$; and $x_{0}$ is an $X$-valued $\Im_{0}$ measurable random variable.

For any Banach space $K$, let $L_{2}(\Omega, K)$ denote the space of strongly measurable $K$ valued square integrable random variables equipped with the norm topology $\|\xi\|_{L_{2}(\Omega, K)} \equiv\left(E\left(\|\xi\|_{K}^{2}\right)\right)^{1 / 2}$, where $E$ stands for integration with respect to the probability 
measure $P$. This is a Hilbert space if $K$ is Hilbert. An $\Im_{t}$-adapted $F$-valued ( $F$ Hilbert) Brownian motion is said to be cylindrical if its covariance operator $Q$, given by

$$
E\left\{e^{-i(W(t), f)}\right\}=e^{-(t / 2)(Q f, f)},
$$

is an identity operator in $F$.

In other words, $E\left\{\left\{^{-i(W(t), f)}\right\}=\exp -(t / 2)\|f\|_{F}^{2}\right.$.

For convenience we shall use $L_{2}^{0}(\Omega, K)$ to denote the class of $K$-valued $\Im_{0}$-measurable square integrable random variables. Let $M(I, K)$ denote the space of $\Im_{t}$-adapted stochastic processes defined on $I$, taking values in $K$, having square integrable norms and continuous in $t$ on $I$ in the mean square sense. This is a Banach space with respect to the norm topology

$$
\|\xi\|_{M(I, K)} \equiv\left(\sup _{t \in I}\left\{E\left(\|\xi(t)\|_{K}^{2}\right)\right\}\right)^{1 / 2} \text {, for } \xi \in M(I, K) .
$$

If $-A$ is the infinitesimal generator of a $C_{0}$-semigroup $T(t), t \geq 0$, in $X$ then the problem (2.1) can be reformulated as a stochastic integral equation,

$$
x(t)=T(t) x_{0}+\int_{0}^{t} T(t-s) f(x(s)) d s+\int_{0}^{t} T(t-s) \sigma(x(s)) d W(s) .
$$

Theorem 6: $\quad$ Suppose the following assumptions hold:

$\left(a_{1}\right): \quad-A$ is the infinitesimal generator of an analytic semigroup $T(t), t \geq 0$, in the Hilbert space $X$.

$\left(a_{2}\right): \quad 0 \leq \alpha<(1 / 2) ; X_{\alpha}=\left[D\left(A^{\alpha}\right)\right]$ is the Banach space with respect to the graph topology induced by the graph norm given by $\|\zeta\|_{\alpha} \equiv\left\|A^{\alpha} \zeta\right\|+\|\zeta\|$ for $\zeta \in D\left(A^{\alpha}\right)$.

$\left(a_{3}\right)$ : The function $f$ maps $X_{\alpha}$ to $X$ and there exists a constant $C>0$ such that,

$$
\begin{gathered}
\|f(\xi)-f(\zeta)\|_{X} \leq C\|\xi-\zeta\|_{\alpha} \text { and } \\
\|f(\zeta)\|_{X} \leq C\left(1+\|\zeta\|_{\alpha}\right) \text { for all } \xi, \zeta \in X_{\alpha} .
\end{gathered}
$$

$\left(a_{4}\right): \sigma$ maps $X_{\alpha}$ to $\mathcal{L}(F, X)$ and there exists a constant $C>0$ such that

$$
\begin{gathered}
\|\sigma(\xi)-\sigma(\zeta)\|_{\mathcal{L}(F, X)} \leq C\|\xi-\zeta\|_{\alpha} \text { and } \\
\|\sigma(\zeta)\|_{\mathcal{L}(F, X)} \leq C\left(1+\|\zeta\|_{\alpha}\right)
\end{gathered}
$$

Then, for every $x_{0} \in L_{2}^{0}\left(\Omega, X_{\alpha}\right)$ and $W$ an $F$-valued $\Im_{t}$-adapted Brownian motion having a nuclear covariance operator $Q \in \mathcal{L}_{n}^{+}(F)$, the integral equation (2.2) has a unique solution $x \in M\left(I, X_{\alpha}\right)$. 
Proof: We use Banach fixed point theorem for the proof. Define the operator $G$ by the expression on the right hand side of equation (2.2) and denote by $z_{1}, z_{2}$, and $z_{3}$ the first, second and the third terms respectively giving $G x \equiv z_{1}+z_{2}+z_{3}$. First we show that $G$ maps $M\left(I, X_{\alpha}\right)$ into $M\left(I, X_{\alpha}\right)$. Without loss of generality we assume that $0 \in \rho(A)$ (if not add a term $\nu I$ to $A$ giving $A_{\nu} \equiv A+\nu I$ so that $\left.0 \in \rho\left(A_{\nu}\right)\right)$ thereby simplifying the graph norm to $\|\zeta\|_{\alpha} \equiv\left\|A^{\alpha} \zeta\right\|$ for $\zeta \in D\left(A^{\alpha}\right)$. Since $T(t), t \geq 0$, is a semigroup and $I$ is a finite interval, there exists a number $M \geq 1$ so that $\sup _{t \in I}\|T(t)\|_{\mathcal{L}(X)} \leq M$. Thus, for $x \in M\left(I, X_{\alpha}\right)$ with $x(0)=x_{0}$, we have

$$
\begin{gathered}
\sup _{t \in I} E\left(\left\|z_{1}(t)\right\|_{\alpha}^{2}\right)=\sup _{t \in I} E\left(\left\|T(t) x_{0}\right\|_{\alpha}^{2}\right)=\sup _{t \in I} E\left(\left\|A^{\alpha} T(t) x_{0}\right\|_{X}^{2}\right) \\
\leq M^{2} E\left(\left\|x_{0}\right\|_{\alpha}^{2}\right) .
\end{gathered}
$$

Since $A^{\alpha}$ is a closed operator and $T(t), t \geq 0$ is an analytic semigroup satisfying $\left\|A^{\alpha} T(t)\right\|_{\mathcal{L}(X)} \leq C_{\alpha} t^{-\alpha}$ for $t>0$, it follows from $\left(a_{3}\right)$ that

$$
\begin{gathered}
E\left(\left\|z_{2}(t)\right\|_{\alpha}^{2}\right)=E\left\|\int_{0}^{t} T(t-s) f(x(s)) d s\right\|_{\alpha}^{2} \\
=E\left\|\int_{0}^{t} A^{\alpha} T(t-s) f(x(s)) d s\right\|_{X}^{2} \\
\leq\left(\int_{0}^{t}\left\|A^{\alpha} T(t-s)\right\|^{2} \mathcal{L}(X) d s\right) E \int_{0}^{t}\|f(x(s))\|_{X}^{2} d s \\
\leq\left(\left(C_{\alpha} C\right)^{2} /(1-2 \alpha)\right) t^{2(1-\alpha)}\left(1+\sup _{0 \leq s \leq t} E\|x(s)\|_{\alpha}^{2}\right) .
\end{gathered}
$$

Hence

$$
\sup _{t \in I}\left(E\left\|z_{2}(t)\right\|_{\alpha}^{2}\right) \leq\left(\left(C_{\alpha} C\right)^{2} /(1-2 \alpha)\right) a^{2(1-\alpha)}\left(1+\|x\|_{M\left(I, X_{\alpha}\right)}^{2}\right)
$$

Similarly, for the stochastic integral $z_{3}$ based on the Brownian motion $W$, it follows from $\left(a_{4}\right)$ that

$$
\begin{aligned}
& E\left(\left\|z_{3}(t)\right\|_{\alpha}^{2}\right) \leq \operatorname{Tr} Q \int_{0}^{t} E\left(\left\|A^{\alpha} T(t-s) \sigma(x(s))\right\|^{2} \mathcal{L}(F, X)\right) d s \\
& \leq \operatorname{Tr} Q\left(2\left(C_{\alpha} C\right)^{2} /(1-2 \alpha)\right) t^{(1-2 \alpha)}\left(1+\underset{0 \leq s \leq t}{\sup _{0 \leq s}} E\|x(s)\|_{\alpha}^{2}\right) .
\end{aligned}
$$

Hence

$$
\sup _{t \in I} E\left(\left\|z_{3}(t)\right\|_{\alpha}^{2}\right) \leq \operatorname{Tr} Q\left(2\left(C_{\alpha} C\right)^{2} /(1-2 \alpha)\right) a^{(1-2 \alpha)}\left(1+\|x\|_{M\left(I, X_{\alpha}\right)}^{2}\right)
$$

where $\operatorname{Tr} Q$ represents the trace of the operator $Q$. 
It follows from $(2.3)-(2.5)$ that

$$
\sup _{t \in I}\|(G x)(t)\|_{\alpha}^{2}<\infty \text { for } x \in M\left(I, X_{\alpha}\right) .
$$

To complete the proof that $G$ maps $M\left(I, X_{\alpha}\right)$ to $M\left(I, X_{\alpha}\right)$, it remains to show that $z \in C\left((0, a), L_{2}\left(\Omega, X_{\alpha}\right)\right)$. Let $t \in(0, a), h>0$ and $t+h \in I \equiv[0, a]$. For analytic semigroups, there exists a constant $\gamma_{\beta}>0$ such that

$$
\|(T(h)-I) \xi\|_{X} \leq \gamma_{\beta} h^{\beta}\left\|A^{\beta} \xi\right\|_{X} \text { for all } \xi \in D\left(A^{\beta}\right) ;
$$

and, for all $\beta \geq 0$ and $\zeta \in X, T(t) \zeta \in D\left(A^{\beta}\right)$ for $t>0$; (see Pazy [10], Theorem 6.13, p. 74). Thus, for $t>0$, one has

$$
\begin{gathered}
E\left(\left\|z_{1}(t+h)-z_{1}(t)\right\|_{\alpha}^{2}\right)=E\left(\left\|(T(h)-I) T(t) A^{\alpha} x_{0}\right\|^{2}\right) \\
\leq \gamma_{\beta}^{2} h^{2 \beta}\left\|A^{\beta} T(t)\right\|^{2} E\left\|A^{\alpha} x_{0}\right\|^{2} \\
\leq\left(\left(\gamma_{\beta} C_{\beta}\right) / t^{\beta}\right)^{2} h^{2 \beta} E\left\|x_{0}\right\|_{\alpha}^{2} .
\end{gathered}
$$

By virtue of closedness of $A^{\alpha}$ and the fact that $T(t)$ commutes with $A^{\alpha}$ on $D\left(A^{\alpha}\right)$ we have,

$$
\begin{gathered}
A^{\alpha}\left(z_{2}(t+h)-z_{2}(t)\right)=\int_{0}^{t}(T(h)-I) A^{\alpha} T(t-s) f(x(s)) d s \\
+\int_{t}^{t+h} A^{\alpha} T(t+h-s) f(x(s)) d s .
\end{gathered}
$$

Choosing $\beta>0$, such that $0 \leq \alpha+\beta<(1 / 2)$, we have $P$-a.s.

$$
\begin{gathered}
\left\|z_{2}(t+h)-z_{2}(t)\right\|_{\alpha} \leq \gamma_{\beta} C_{\alpha+\beta^{h^{\beta}}} \int_{0}^{t}\left(1 /(t-s)^{\alpha+\beta}\right)\|f(x(s))\| d s \\
+C_{\alpha} \int_{t}^{t+h}\left(1 /(t+h-s)^{\alpha}\right)\|f(x(s))\| d s .
\end{gathered}
$$

Hence, using $\left(a_{3}\right)$ and Schwartz inequality, one can find constants $C_{1}$ and $C_{2}$ depending on the parameters $a, C, \alpha, \beta, \gamma_{\beta}, C_{\alpha}$, and $C_{\alpha+\beta}$ such that

$$
E\left\|z_{2}(t+h)-z_{2}(t)\right\|_{\alpha}^{2} \leq\left(C_{1} h^{2 \beta}+C_{2} h^{2(1-\alpha)}\right)\left(1+\sup _{s \in I} E\|x(s)\|_{\alpha}^{2}\right)
$$

for $t \in(0, a)$. Similarly, for the stochastic integral $z_{3}$, using $\left(a_{4}\right)$, one can find constants $c_{3}$ and $c_{4}>0$, such that 


$$
E\left\|z_{3}(t+h)-z_{3}(t)\right\|_{\alpha}^{2} \leq \operatorname{Tr} Q\left(C_{3} h^{\beta}+C_{4} h^{(1-2 \alpha)}\right)\left(1+\sup _{s \in I} E\|x(s)\|_{\alpha}^{2}\right)
$$

for $t \in(0, a)$. Similar estimates hold for $E\|z(t-h)-z(t)\|_{\alpha}^{2}$ for $t \geq h>0$. Thus letting $h \rightarrow 0$, the desired continuity follows from $(2.7)-(2.9)$ and hence $G$ maps $M\left(I, X_{\alpha}\right)$ to $M\left(I, X_{\alpha}\right)$. Now we prove that, for sufficiently small $a$ defining the interval $I \equiv[0, a], G$ is a contraction in $M\left(I, X_{\alpha}\right)$. Indeed, for $x, y \in M\left(I, X_{\alpha}\right)$ satisfying $x(0)=y(0)=x_{0} P$-a.s., using $\left(a_{3}\right)$ and $\left(a_{4}\right)$, one can easily verify that

$$
\sup _{t \in I} E\|(G x)(t)-(G y)(t)\|_{\alpha}^{2} \leq K_{a} \sup _{t \in I} E\|x(t)-y(t)\|_{\alpha}^{2}
$$

where

$$
K_{a} \equiv\left(2\left(C C_{\alpha}\right)^{2} /(1-2 \alpha)\right)\left(a^{2(1-\alpha)}+\operatorname{Tr} Q a^{(1-2 \alpha)}\right) .
$$

Thus, for sufficiently small $a, K_{a}<1$ and $G$ is a contraction in $M\left(I, X_{\alpha}\right)$ and hence, by Banach fixed point theorem, $G$ has a unique fixed point $x \in M\left(I, X_{\alpha}\right)$. Clearly, by virtue of the growth conditions in $\left(a_{3}\right)$ and $\left(a_{4}\right)$ and the continuity and uniqueness, the solution can be continued indefinitely by piecing together the solutions obtained for the intervals $(0, a],(a, 2 a]$, $(2 a, 3 a]$ and so on. Thus, for any finite interval $I$, the integral equation (2.2) has a unique solution $x \in M\left(I, X_{\alpha}\right)$ which is the mild solution of the stochastic evolution equation (2.1).

Remark 7: This result can be easily localized and further, if $-A(t)$ is the generator of an evolution operator of "parabolic type", it can be extended to cover time varying systems. In equation (2.1) one can also include an integral term (representing memory) without further complication.

The result of Theorem 4 can be extended to stochastic problems as stated in the following theorem.

Theorem 8: $\quad$ Consider the stochastic evolution equation,

$$
\begin{gathered}
d x+A(t) x d t=f(t, x) d t+\sigma(t, x) d W, t \in I \equiv[0, a], a<\infty, \\
x(0)=x_{0},
\end{gathered}
$$

and suppose $A$ satisfies the assumptions of Lemma 1 , and $f$ maps $I \times H \rightarrow V^{-\alpha}$ and $\sigma$ maps $I \times H \rightarrow L\left(H, V^{-\alpha}\right)$ for $0 \leq \alpha<1$ satisfying the following conditions: there exists a constant $K>0$ such that

$$
\begin{gathered}
(f): \quad\|f(t, \zeta)\|_{V}^{2}-\alpha \leq K^{2}\left(1+\|\zeta\|_{H}^{2}\right),\|f(t, \zeta)-f(t, \xi)\|_{V}^{2}-\alpha \leq K^{2}\|\zeta-\xi\|_{H}^{2}, \\
\left.(\sigma): \quad\|\sigma(t, \zeta)\|^{2}{ }_{\mathcal{L}(H, V}^{-\alpha}\right) \leq K^{2}\left(1+\|\zeta\|_{H}^{2}\right), \\
\left.\|\sigma(t, \zeta)-\sigma(t, \xi)\|_{\mathcal{L}(H, V}^{2}{ }^{2}\right) \leq K^{2}\|\zeta-\xi\|_{H}^{2}
\end{gathered}
$$


Then, for every $x_{0} \in L_{2}^{0}(\Omega, H)$ and Wiener process $W$ with covariance operator $Q \in \mathcal{L}_{n}^{+}(H)$, the equation (2.11) has a mild solution $x \in M(I, H)$ given by the solution of the integral equation

$$
x(t)=U(t, 0) x_{0}+\int_{0}^{t} U(t, s) f(s, x(s)) d s+\int_{0}^{t} U(t, s) \sigma(s, x(s)) d W(s) .
$$

Proof: The proof is based on Banach fixed point theorem that sues the following inequality similar to (1.14),

$$
E\|(G x)(t)-(G y)(t)\|_{H}^{2} \leq \gamma \int_{0}^{t}\left(1 /(t-s)^{\alpha}\right) E\|x(s)-y(s)\|_{H}^{2} d s
$$

where $\gamma$ is a constant depending on $a, c, K$ and $q \equiv \operatorname{Tr} Q$.

Remark 9: In Theorem 6, if the diffusion operator $\sigma(t, x)$ is taken as zero one can admit $\alpha \in[0,1)$ as in Theorem 5. Similarly, note that, in Theorem $8, \alpha$ cannot take the value 1 , that is, neither $f$ nor $\sigma$ can be as singular as in Theorem 4 where range $(f)$, range $(\sigma) \subseteq V^{*}=V^{-1}$. These results show that in the stochastic case the nonlinear operators $f$ and $\sigma$ have to be much more regular compared to their deterministic counterparts.

Next we consider a general class of stochastic quasilinear evolution equations given by,

$$
\begin{gathered}
d x=A(t, x) x d t+\sigma(t) d W, t \in I \equiv[0, a], \\
x(0)=x_{0}
\end{gathered}
$$

in a Hilbert space $X$ considered as the state space where $\{W(t), t \geq 0\}$ is an $\Im_{t}$-Brownian motion taking values in a separable Hilbert space $F$. The generality comes from the assumptions on the operator $A(t, x)$. Here we assume that for each $(t, \xi) \in I \times X, A(t, \xi)$ is the generator of a $C_{0}$-semigroup rather than an analytic semigroup. The deterministic version of equation (2.14) which has broad applications in engineering and physical sciences was studied by Kato [7,8]; Pazy [10].

For simplicity of presentation we introduce the notation $G(Z, M, \omega)$ to denote the class of infinitesimal generators $\{A\}$ of $C_{0}$-semigroups $\left\{T_{A}(t), t \geq 0\right\}$ in any Banach space $Z$ satisfying $\left\|T_{A}(t)\right\|_{\mathcal{L}(Z)} \leq M$ exp $\omega t$, for $t \geq 0$, where $M \geq 1$, and $\omega \in R$ are the stability parameters. We use the following basic assumptions:

$\left(A_{1}\right)$ : There exists a Hilbert space $Y$ with the embedding $Y \hookrightarrow X$ being continuous and dense. $\left(A_{2}\right)$ : For each $t \in I$ and $\xi \in X, A(t, \xi) \in \mathfrak{g}(X, M, \omega)$ and $Y$ is $A(t, \xi)$ admissible in the sense 
that $T_{A}(\tau) Y \subseteq Y$ for $\tau \geq 0$, where $T_{A}(\tau)$ is the semigroup corresponding to $A(t, \xi)$. Further $\tilde{A}(t, \xi) \in \mathcal{g}(Y, \tilde{M}, \tilde{\omega})$, where $\tilde{A}(t, \xi)$ is the part of $A(t, \xi)$ with domain and range in $Y$ and $\tilde{M}, \tilde{\omega}$ the associated stability parameters.

$\left(A_{3}\right)$ : For each $t \in I$ and $\xi \in X, D(A(t, \xi)) \supseteq Y$ and $A(t, \xi) \in \mathcal{L}(Y, X)$.

$\left(A_{4}\right)$ : For each $\xi \in X, t \rightarrow A(t, \xi)$ is continuous in the uniform operator topology of $\mathcal{L}(Y, X)$; and there exists a constant $K>0$, independent of $t$ such that

$$
\|A(t, \xi)-A(t, \zeta)\|_{\mathcal{L}(Y, X)} \leq K\|\xi-\zeta\|_{X} \text { for all } t \in I .
$$

These assumptions are somewhat stronger than those given for deterministic systems (see Kato $[7,8])$. It appears that for stochastic systems this is unavoidable.

Theorem 10: Consider the quasilinear system (2.11) satisfying the hypotheses $\left(A_{1}\right)-\left(A_{4}\right)$ and suppose $\sigma \in L_{2}(I, \mathcal{L}(F, Y))$ and there exists a nuclear operator $Q \in \mathcal{L}_{n}^{+}(F)$ so that $E(v, W(t)-W(s))^{2}=(t-s)(Q v, v)$ for each $v \in F$ and $t \geq s$. Then for every $x_{0} \in L_{\infty}^{0}(\Omega, Y)$ there exists an $a^{*} \in(0, a]$ such that the system (2.11) has a unique mild solution $x \in M\left(\left[0, a^{*}\right], X\right)$.

Proof: $\quad$ For convenience, we use $M_{a}$ to denote the Banach space $M([0, a], X)$, as defined in the introduction preceding Theorem 6. Take any $y \in M_{a}$ and consider the linear evolution equation,

$$
\begin{gathered}
d \xi=A(t, y(t)) \xi d t+\sigma(t) d W(t), t \in I \equiv[0, a], \\
\xi(0)=x_{0} .
\end{gathered}
$$

Define $A^{y}(t) \equiv A(t, y(t)), t \in I$. Under the assumptions $\left(A_{1}\right)-\left(A_{4}\right)$ the operator $A^{y}$ generates an evolution operator $U^{y}(t, x), 0 \leq s \leq t \leq a$, (see Kato [7], Theorem 4.1, p. 246; Pazy [10], Theorem 4.3, p. 202). Then by virtue of the variation of constants formula, we may define the mapping $G$ by

$$
\xi(t)=(G y)(t) \equiv U^{y}(t, 0) x_{0}+\int_{0}^{t} U^{y}(t, s) \sigma(s) d W(s),
$$

for $t \in I$. From the almost sure strong continuity of $U^{y}(t, s)$ on the triangle $0 \leq s \leq t \leq a$ and the fact that, for each $\Im_{s}$-measurable random variable $\eta, U^{y}(t, s) \eta$ is $\Im_{t}$ measurable and $W(t)$ is $\Im_{t}$-adapted it follows that $\xi(t)$ is $\Im_{t}$-adapted. Hence from similar computations as in the preceding theorem, we have $G y \in M_{a}$. So it suffices to prove that, for sufficiently small $a$, the operator $G$ is a contraction in $M_{a}$. Let $x, z \in M_{a}$ satisfying $x(0)=z(0)=x_{0} P$-a.s. First, note that for $0 \leq s \leq \tau \leq t \leq a$ and $\xi \in Y$, 


$$
\left(\partial^{+} / \partial \tau\right)\left(U^{x}(t, \tau) U^{z}(\tau, s) \xi\right)=U^{x}(t, \tau)\left(A^{z}(\tau)-A^{x}(\tau)\right) U^{z}(\tau, s) \xi
$$

$P-a . s$. and hence integrating this over the integral $[s, t]$ we obtain

$$
U^{z}(t, s) \xi-U^{x}(t, s) \xi=\int_{s}^{t} U^{x}(t, \tau)\left(A^{z}(\tau)-A^{x}(\tau)\right) U^{z}(\tau, s) \xi d \tau
$$

Now letting $J_{1}$ and $J_{2}$ denote the first and the second terms of the following expression,

$$
\begin{gathered}
(G x)(t)-(G z)(t)=\left(U^{x}(t, 0)-U^{z}(t, 0)\right) x_{0}+\int_{0}^{t}\left(U^{x}(t, s)-U^{z}(t, s)\right) \sigma(s) d W \\
\equiv J_{1}(t)+J_{2}(t)
\end{gathered}
$$

it follows from (2.14) and assumptions $\left(A_{2}\right)-\left(A_{4}\right)$ that

$$
\begin{gathered}
\left\|J_{1}(t)\right\|_{X}=\left\|\int_{0}^{t} U^{z}(t, s)\left(A^{x}(s)-A^{z}(s)\right) U^{x}(s, 0) x_{0} d s\right\|_{X} \\
\leq\left\|x_{0}\right\|_{Y} \int_{0}^{t}\left\|U^{z}(t, s)\right\|_{\mathcal{L}(X)}\left\|A^{x}(s)-Z^{z}(s)\right\|_{\mathcal{L}(Y X)}\left\|U^{x}(s, 0)\right\|_{\mathcal{L}(Y)} d s \\
\leq C\left\|x_{0}\right\|_{Y} \int_{0}^{t}\|x(s)-z(s)\|_{X} d s
\end{gathered}
$$

where $C \equiv K M \tilde{M} \exp \gamma a$ with $\gamma \equiv \max \{\omega, \tilde{\omega}, 0\}$. Hence

$$
E\left(\left\|J_{1}(t)\right\|^{2}\right) \leq C^{2} t\left(\text { ess.sup }\left\|x_{0}\right\|_{Y}^{2}\right)\left(E \int_{0}^{t}\|x(s)-z(s)\|_{X}^{2} d s\right) .
$$

By use of the nuclearity of the operator $Q$, one can easily verify that the stochastic term $J_{2}$ satisfies the following estimate

$$
\begin{gathered}
E\left(\left\|J_{2}(t)\right\|_{X}\right)^{2} \leq \operatorname{Tr} Q \int_{0}^{t} E\left\|\sigma^{*}(s)\left(U^{x}(t, s)-U^{z}(t, s)\right)^{*}\right\|^{2} \mathcal{L}(Y, X) d s \\
\leq \operatorname{Tr} Q \int_{0}^{t}\|\sigma(s)\|^{2}{ }_{\mathcal{L}(F, Y)} E\left\|U^{x}(t, s)-U^{z}(t, s)\right\|^{2}{ }_{\mathcal{L}(Y, X)} d s .
\end{gathered}
$$

By virtue of assumption $\left(A_{4}\right)$ it follows from (2.17) that

$$
E\left\|U^{x}(t, s)-U^{z}(t, s)\right\|_{\mathcal{L}(Y, X)}^{2} \leq(C K)^{2}(t-s) \int_{S}^{t} E\|x(\tau)-z(\tau)\|_{X}^{2} d \tau,
$$

where the constants $C, K$ are as defined earlier. Hence 


$$
E\left\|J_{2}(t)\right\|_{X}^{2} \leq \operatorname{Tr} Q(C K)^{2}\left(\int_{0}^{t}\|\sigma(\nu)\|_{\mathcal{L}(F, Y)}^{2} d \nu\right)\left(\int_{0}^{t} E\|x(s)-z(s)\|_{X}^{2} d s\right)
$$

for $t \in I$. Defining

$$
K_{1} \equiv C^{2}\left\|x_{0}\right\|_{L_{\infty}(\Omega, Y)}^{2} \text { and } K_{2} \equiv \operatorname{Tr} Q(C K)^{2} \int_{0}^{a}\|\sigma(s)\|^{2} \mathcal{L}(F, Y) d s
$$

from $(2.16)-(2.20)$ that

$$
\|G x-G z\|_{M_{a}} \leq a \sqrt{\left(K_{1}+K_{2}\right)}\|x-z\|_{M_{a}} .
$$

Hence there exists a constant $a^{*}$, as stated in the theorem, for which $G$ is a contraction in $M_{a^{*}}$, thereby proving the theorem.

Remark 11: In system (2.14) we can easily include a nonlinear drift term $f(t, x)$ without further complication provided it is more regular than the principal part. However, if one wishes to admit nonlinear diffusion $\sigma(t, x)$, it is required that $\sigma$ be uniformly bounded on $I \times X$. Under the given assumptions on the quasilinear term it seems it is unavoidable.

The result of Theorem 10 can be extended to the case where $F$ is a separable Banach space, $Q \in \mathcal{L}_{n}^{+}\left(F^{*}, F\right)$, and $X$ is a Banach space having a separable dual.

For semilinear stochastic systems see DaPrato, Iannelli, Tubaro [6]. Some results on deterministic and stochastic initial boundary value problems based on the theory of monotone and accretive operators and semigroup theory can be found in [2], [3], [4].

\section{REFERENCES}

[1] N.U. Ahmed, "Nonlinear integral equations on reflexive Banach spaces with applications to stochastic integral equations and abstract evolution equations", J. of Integral Equations, Vol. 1, (1979), pp. 1-15.

[2] N.U. Ahmed, "Semigroup theory with applications to systems and control", Pitman Research Notes in Mathematics series, Vol. 246, (1991), Longman Scientific and Technical, U.K.

[3] N.U. Ahmed, "Stochastic initial-boundary value problems for a class of second order evolution equations", Proc. of International Conf. on Theory and Applications of Differential Equations, (ed. A.R. Aftabizadeh), Vol. 1, Ohio University Press, (1988), pp. 13-19.

[4] N.U. Ahmed, "Optimization and identification of systems governed by evolution equations on Banach space", Pitman Research Notes in Mathematics series, Vol. 184, (1988), Longman Scientific and Technical, U.K. 
[5] G. DaPrato, "Abstract differential equations, maximal regularity, and linearization, nonlinear functional analysis and its applications", Proc. Symp. Pure Math AMS, Vol. 45, Part 1, (1986), pp. 359-370.

[6] G. DaPrato, M. Iannelli, and L. Tubaro, "Semilinear stochastic differential equations on Hilbert spaces”, Bollettino Unione Matematica Italiana, 5 16-A, (1978), pp. 168-185.

[7] T. Kato, "Linear evolution equations of 'hyperbolic' type", J. Fac. Sci. Univ. Tokyo, Sec. 1, (1970), pp. 241-258.

[8] T. Kato, "Linear evolution equations of 'hyperbolic' type, II", J. Math. Soc., Japan, Vol. 25, 4, (1973), pp. 648-666.

[9] G.E. Ladas and V. Lakshmikantham, "Differential equations in abstract spaces", Math. in Science and Engineering, Vol. 85, Academic Press, (1972).

[10] A. Pazy, "Semigroups of linear operators and applications to partial differential equations", App. Math. Sci., Vol. 44, Springer-Verlag, New York, Berlin, Heidelberg, Tokyo, (1983).

[11] H. Tanabe, “Equations of Evolutions", Pitman Monographs and Studies in Mathematics, Vol. 6, Pitman, London, San Francisco, Melbourne, (1979). 


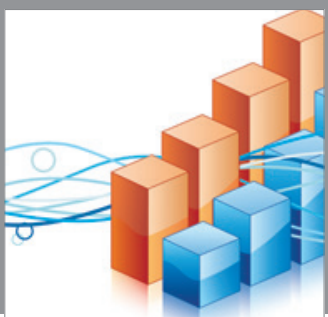

Advances in

Operations Research

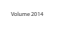

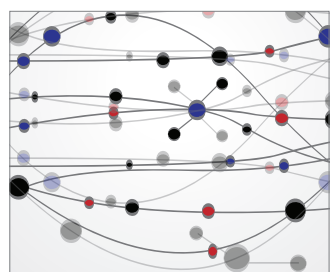

\section{The Scientific} World Journal
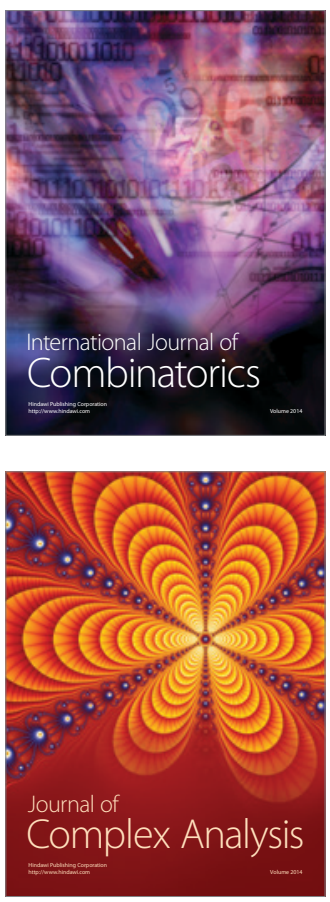

International Journal of

Mathematics and

Mathematical

Sciences
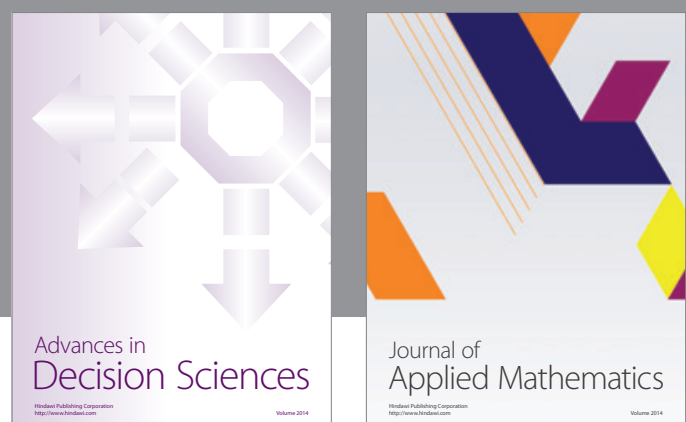

Journal of

Applied Mathematics
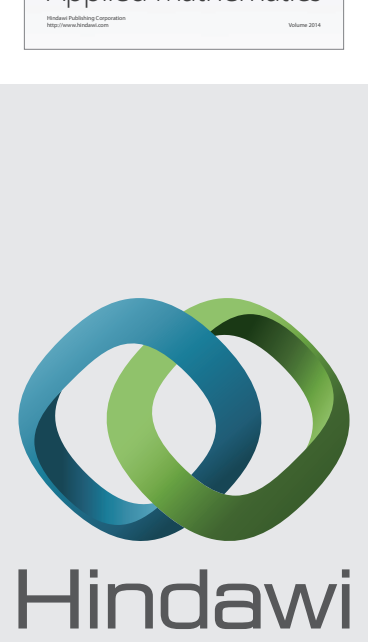

Submit your manuscripts at http://www.hindawi.com
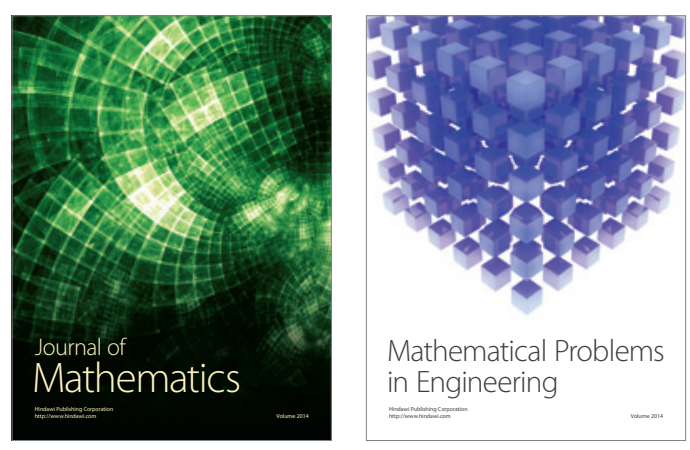

Mathematical Problems in Engineering
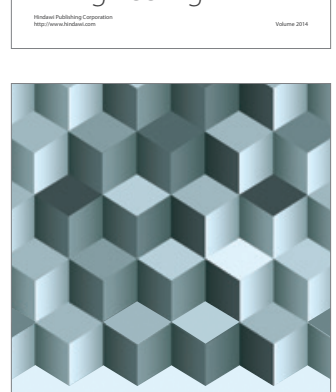

Journal of

Function Spaces
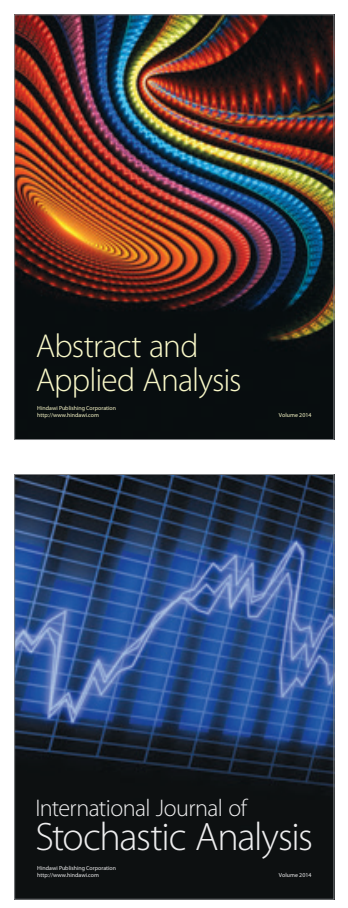

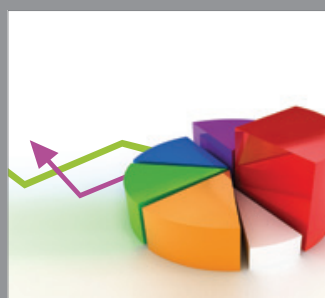

ournal of

Probability and Statistics

Promensencen
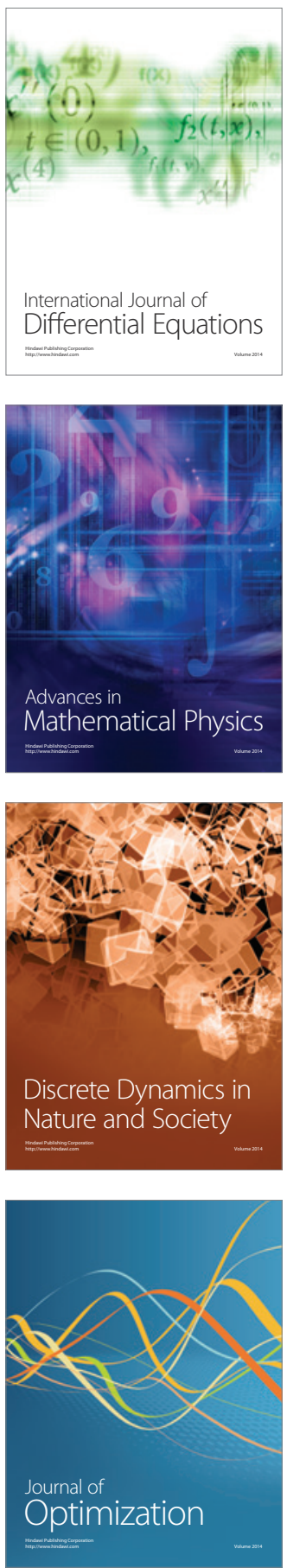